\title{
Author Correction: A novel WEE1 pathway for replication stress responses
}

Ting Pan, Qi Qin, Chubing Nong, Shan Gao, Lili Wang, Bingcheng Cai, Ming Zhang, Chong Wu, Hanchen Chen, Tong Li, Dan Xiong, Guoliang Li (1), Shui Wang (D) and Shunping Yan (i)

Correction to: Nature Plants https://doi.org/10.1038/s41477-021-00855-8, published online 11 February 2021.

In Fig. 6e of the Article originally published, the downwards-pointing arrow between CKIs and CDKs should have been a blunted arrow. This error has now been corrected.

Published online: 24 February 2021

https://doi.org/10.1038/s41477-021-00883-4

(c) The Author(s), under exclusive licence to Springer Nature Limited 2021 